\title{
Optimal control problems for affine connection control systems: characterization of extremals.
}

\author{
M. Barbero-Liñán and M.C. Muñoz-Lecanda \\ Departamento de Matemática Aplicada IV, Edificio C-3, Campus Norte UPC. C/ Jordi Girona 1. \\ E-08034 Barcelona. Spain. \\ mbarbero@ma4.upc.edu,matmcml@ma4.upc.edu
}

\begin{abstract}
Pontryagin's Maximum Principle [8] is considered as an outstanding achievement of optimal control theory. This Principle does not give sufficient conditions to compute an optimal trajectory; it only provides necessary conditions. Thus only candidates to be optimal trajectories, called extremals, are found. Maximum Principle gives rise to different kinds of them and, particularly, the so-called abnormal extremals have been studied because they can be optimal, as Liu and Sussmann, and Montgomery proved in subRiemannian geometry [5, 7].

We build up a presymplectic algorithm, similar to those defined in $[2,3,4,6]$, to determine where the different kinds of extremals of an optimal control problem can be. After describing such an algorithm, we apply it to the study of extremals, specially the abnormal ones, in optimal control problems for affine connection control systems [1]. These systems model the motion of different types of mechanical systems such as rigid bodies, nonholonomic systems and robotic arms [1].
\end{abstract}

Keywords: Pontryagin's Maximum Principle, abnormal extremals, optimal control problems, affine connection control systems.

PACS: $02.30 Y y, 45.80 .+r, 46.15 . C c, 02.40 Y y, 02.30 X x$

\section{OPTIMAL CONTROL PROBLEM FOR AFFINE CONNECTION CONTROL SYSTEMS}

Let $Q$ be a smooth $n$-dimensional manifold, $\nabla$ be an affine connection on $Q, U$ be an open set in $\mathbb{R}^{m}$. We consider the control system with dynamics given by

$$
\nabla_{\dot{\gamma}(t)} \dot{\gamma}(t)=u^{k}(t) Y_{k}(\gamma(t)), \quad k=1, \ldots, m,
$$

where the controls $u: I \subset \mathbb{R} \rightarrow U \subset \mathbb{R}^{m}$ are piecewise smooth, $Y_{k}$ are the input vector fields on $Q$. This control system is called an Affine Connection Control System, ACCS, $\Sigma=(Q, \nabla, \mathscr{Y}, U)$ where $\mathscr{Y}=\left\{Y_{1}, \ldots, Y_{m}\right\}$.

In fact, the affine connection control systems are a particular case of the general simple mechanical systems without external forces nor nonholonomic constraints [1].

The second-order equation (1) is rewritten on $T Q$ as follows,

$$
\dot{\Upsilon}(t)=Z(\Upsilon(t))+u^{k}(t) Y_{k}^{V}(\Upsilon(t))
$$

where $\Upsilon: I \rightarrow T Q$ is a piecewise smooth curve such that $\Upsilon=\dot{\gamma}, Y_{k}^{V}$ denotes the vertical lift of the vector field $Y_{k}$ and the drift vector field $Z$ of the control-affine system (2) is the geodesic spray associated to $\nabla$. The vector field $Z$ on $T Q$ is second order. In natural coordinates $(x, v)$ for $T Q$, locally $Z=v^{i} \partial / \partial x^{i}-\Gamma_{j l}^{i}(x) v^{j} v^{l} \partial / \partial v^{i}$ with Christoffel symbols $\Gamma_{j l}^{i}$ for $\nabla$. 
Let $F: T Q \times U \rightarrow \mathbb{R}$ be a cost function. We study the following problem.

Problem 1. (Free-time Optimal Control Problem for ACCS (OCP)) Given $\Sigma=(Q, \nabla, \mathscr{Y}, U)$ and $F$, find $I=[a, b] \subset \mathbb{R}$ and $(\gamma, u): I \rightarrow Q \times U$ such that, given the endpoint conditions $v_{x_{a}} \in T_{x_{a}} Q, v_{x_{b}} \in T_{x_{b}} Q$, there exists $\Upsilon: I \rightarrow T Q$ along $\gamma$ satisfying

(1) $\Upsilon(a)=v_{x_{a}}, \Upsilon(b)=v_{x_{b}}$,

(2) $\dot{\Upsilon}(t)=\left(Z+u^{k} Y_{k}^{V}\right)(\Upsilon(t))$,

(3) $\mathscr{S}[\Upsilon, u]=\int_{I} F(\Upsilon(t), u(t)) d t$ minimizes over all curves on $T Q \times U$ satisfying $(1,2)$.

The pair $(\Upsilon, u)$ is called a solution of OCP.

The Hamiltonian of Pontryagin's Maximum Principle, Theorem 1, for the controlaffine system (2) on $T Q$ is given by $H: T^{*}(T Q) \times U \rightarrow \mathbb{R}$,

$$
H(\Lambda, u)=\left\langle\Lambda, Z\left(v_{x}\right)+u^{k} Y_{k}^{V}\left(v_{x}\right)\right\rangle+p_{0} F\left(v_{x}, u\right)
$$

where $\Lambda \in T_{v_{x}}^{*}(T Q), p_{0} \in\{-1,0\}$ and $\langle\cdot, \cdot\rangle$ is the natural pairing.

Let us introduce some notation. If $X$ is a vector field on a manifold $M$, we associate to it the hamiltonian function $H_{X}: T^{*} M \rightarrow \mathbb{R}$ defined by $\lambda \mapsto H_{X}(\lambda)=\langle\lambda, X(x)\rangle$. Then we can rewrite Pontryagin's Hamiltonian (3) as follows $H=H_{Z}+u^{k} H_{Y_{k}^{V}}+p_{0} F$.

In the sequel, we use a presymplectic framework to state Pontryagin's Maximum Principle because $\left(T^{*}(T Q) \times U, \Omega, H\right)$ is a presymplectic Hamiltonian system with $\Omega$ being the pullback of the canonical 2-form on $T^{*}(T Q)$ through $\pi_{1}: T^{*}(T Q) \times U \rightarrow$ $T^{*}(T Q)$. For more details on presymplectic formalism see $[2,4]$.

Theorem 1. (Weak Pontryagin's Maximum Principle, PMP) Let $(\Upsilon, u):[a, b] \rightarrow T Q \times$ $U$ be a solution of OCP with endpoint conditions $v_{x_{a}}, v_{x_{b}}$. Then there exist $\Lambda:[a, b] \rightarrow$ $T^{*}(T Q)$ along $\Upsilon$, and a constant $p_{0} \in\{-1,0\}$ such that:

1. $(\Lambda, u)$ is an integral curve of a Hamiltonian vector field $X_{H}$ such that

$$
i_{X_{H}} \Omega=\mathrm{d} H \text {, i.e. } i_{(\dot{\Lambda}(t), \dot{u}(t))} \Omega=\mathrm{d} H(\Lambda(t), u(t)) ;
$$

2. $\Upsilon=\pi_{T Q} \circ \Lambda$, where $\pi_{T Q}: T^{*}(T Q) \rightarrow T Q$ is the natural projection;

3. $\Upsilon$ satisfies the endpoint conditions in $T Q$;

4. (a) $H(\Lambda(t), u(t))=0$ for $t \in[a, b]$;

(b) $\left(p_{0}, \Lambda(t)\right) \neq 0$ for each $t \in[a, b]$.

The presymplectic equation (4) does not have solution in the whole manifold $T^{*}(T Q) \times U$. It has solution if we restrict (4) to the submanifold defined by

$$
S=\left\{\beta \in T^{*}(T Q) \times U \mid i_{\nu} \mathrm{d} H=0 \text { for } v \in \operatorname{ker} \Omega_{\beta}\right\} .
$$

Locally, this condition for Pontryagin's Hamiltonian (3) becomes $S=\left\{\beta \in T^{*}(T Q) \times\right.$ $\left.U \mid \partial H / \partial u^{k}(\beta)=0, k=1, \ldots, m\right\}$. Observe that this is a necessary condition for the Hamiltonian to have an extremum over the controls as long as $U$ is an open set. In the classic statement of Pontryagin's Maximum Principle [8], the Hamiltonian along the solutions is equal to the maximum of the Hamiltonian over the controls. Therefore, Theorem 1 is weaker than the classic Maximum Principle. 
The necessary conditions 1-4 of Theorem 1 determine different extremals.

Definition 1. A curve $(\Upsilon, u):[a, b] \rightarrow T Q \times U$ for $O C P$ is

1. an extremal if there exist $\Lambda:[a, b] \rightarrow T^{*}(T Q)$ and a constant $p_{0} \in\{-1,0\}$ such that $\Upsilon=\pi_{T Q} \circ \Lambda$ and $(\Lambda, u)$ satisfies the necessary conditions of weak PMP;

2. a normal extremal if it is an extremal and $p_{0}=-1$;

3. an abnormal extremal if it is an extremal and $p_{0}=0$;

4. a strictly abnormal extremal if it is not a normal extremal, but it is abnormal.

The curve $(\Lambda, u):[a, b] \rightarrow T^{*}(T Q) \times U$ along $\Upsilon$ is called biextremal.

\section{PRESYMPLECTIC CONSTRAINT ALGORITHM FOR ACCS}

The presymplectic algorithm described in $[2,4]$ can be adapted to study the different kinds of extremals for ACCS. First we describe briefly how this classic algorithm works.

Problem 2. Given a presymplectic system $(M, \Omega, H)$ find $(N, X)$ such that

(a) $N$ is a submanifold of $M$,

(b) $X$ is a vector field tangent to $N$ on $N$ and satisfies $i_{X} \Omega=\mathrm{d} H$,

(c) $N$ is maximal among all the submanifolds satisfying $(a)$ and $(b)$.

Let $N_{0}=\left\{x \in M \mid \exists v_{x} \in T_{x} M, i_{v_{x}} \Omega=\mathrm{d}_{x} H\right\}$ be the primary constraint submanifold, where the presymplectic equation has solution. The constraints define $N_{0}$ implicitly.

Proposition 1. $N_{0}=\left\{x \in M \mid\left(\mathrm{L}_{Z} H\right)_{x}=0, Z \in \operatorname{ker} \Omega\right\}$, where $\mathrm{L}_{Z}$ is the Lie derivative with respect to $Z$.

The proof is a straightforward consequence of the fact that if $\alpha_{x} \in T_{x}^{*} M$, we have $\alpha_{x} \in \operatorname{Im} \Omega_{x}^{\sharp}$ if and only if $\operatorname{ker} \Omega_{x} \subset \operatorname{ker} \alpha_{x}$. See [4] for details.

On the points of $N_{0}$ there exists a solution of the presymplectic equation, but the solution is not necessarily unique. Indeed, if $X_{0}$ is a solution, then $X_{0}+\operatorname{ker} \Omega$ is the set of all the solutions. Take the pair $\left(N_{0}, X^{N_{0}}\right)$ where $X^{N_{0}}=X_{0}+\operatorname{ker} \Omega$ denotes the set of all the vector fields solving the problem in $N_{0}$. Observe that we need an element in $X^{N_{0}}$ tangent to $N_{0}$. Then $N_{1}=\left\{x \in N_{0} \mid \exists X \in X^{N_{0}}, X(x) \in T_{x} N_{0}\right\}$ provides us a new pair $\left(N_{1}, X^{N_{1}}\right)$ where $X^{N_{1}}$ is the set of the vector fields solution tangent to $N_{0}$ and we assume again that $N_{1}$ is a submanifold. This step stabilizes the constraints in $N_{0}$. Inductively, we obtain $\left(N_{i}, X^{N_{i}}\right)$ where we assume that $N_{i}$ is a submanifold. If there exists $i \in \mathbb{N}$ such that $N_{i}=N_{i-1}$, then $N_{f}=N_{i-1}$ is the final constraint submanifold.

\subsection{Application to the characterization of extremals}

In order to study OCP for ACCS, we need to solve the presymplectic equation (4) on the submanifold defined by $H=0$. Applying the above algorithm, we obtain

$$
N_{0}=\left\{(\Lambda, u) \in T^{*}(T Q) \times U \mid H=0, \partial H / \partial u^{k}=H_{Y_{k}^{V}}+p_{0} \partial F / \partial u^{k}=0, k=1, \ldots, m\right\} .
$$


The tangency condition of $X_{H}$ to $N_{0}$ defines

$$
N_{1}=\left\{(\Lambda, u) \in N_{0} \mid X_{H}(H)=0, X_{H}\left(\partial H / \partial u^{k}\right)=0, k=1, \ldots, m\right\} .
$$

The first constraint is trivially stabilized because of the properties of Hamiltonian systems. So it may be put aside and be added at the end as is done in Section 2.2.

In the particular case of abnormality, $p_{0}=0$, the submanifold (5) becomes

$$
N_{0}^{[0]}=\left\{(\Lambda, u) \in T^{*}(T Q) \times U \mid H^{[0]}=0, H_{Y_{k}^{V}}=0, k=1, \ldots, m\right\} .
$$

and the stabilization step (6) defines $N_{1}^{[0]}=\left\{(\Lambda, u) \in N_{0}^{[0]} \mid H_{\left[Z, Y_{k}^{V}\right]}=0, k=1, \ldots, m\right\}$. The algorithm must continue to obtain a final constraint submanifold $N_{f}^{[0]}$ where the abnormal biextremals are. Condition (4.c) in Theorem 1 makes us delete the zero fiber from $N_{f}^{[0]}$. For simplicity, this actual final constraint submanifold is renamed as $N_{f}^{[0]}$.

In the case of normality, $p_{0}=-1$, the primary constraint submanifold becomes

$$
N_{0}^{[-1]}=\left\{(\Lambda, u) \in T^{*}(T Q) \times U \mid H^{[-1]}=0, H_{Y_{k}^{V}}-\partial F / \partial u^{k}=0, k=1, \ldots, m\right\} .
$$

Without having specified the cost function, the stabilization procedure gives expressions more and more complicated and we are not going to write them down. The algorithm must continue up to obtaining $N_{f}^{[-1]}$.

As a consequence of both final constraint submanifolds, the strict abnormal extremals are obtained by the projection of all the biextremals in $N_{f}^{[0]}$ and $N_{f}^{[-1]}$ through $\rho: T^{*}(T Q) \times U \rightarrow T Q \times U$. Remember that the strict abnormal extremals can be only lifted to the cotangent bundle with $p_{0}=0$. Denoting by $P=\rho\left(N_{f}^{[0]}\right) \cap \rho\left(N_{f}^{[-1]}\right)$, we have

(i) $P=\emptyset$ and $\rho\left(N_{f}^{[0]}\right) \neq \emptyset$, then all the abnormal extremals are strict.

(ii) $P=\emptyset$ and $\rho\left(N_{f}^{[-1]}\right) \neq \emptyset$, none of the normal extremals are abnormal.

(iii) $P \neq \emptyset$ and $\rho\left(N_{f}^{[0]}\right)=P$, then there are no strict abnormal extremals.

(iv) $P \neq \emptyset$ and $\rho\left(N_{f}^{[0]}\right) \neq P$, there are strict abnormal extremals, but only locally since the extremal could have pieces in $P$. So the extremal can be locally normal.

(v) $P \neq \emptyset$ and $\rho\left(N_{f}^{[0]}\right)=\rho\left(N_{f}^{[-1]}\right)=P$, then all the extremals are abnormal and normal.

\subsection{Time-Optimal Control Problem}

We have a free-time optimal control problem with cost function $F=1$. As the stabilization of the condition $H=0$ is guaranteed trivially, its tangency condition does not add any new constraint to the submanifolds of the algorithm. Then we can run the algorithm putting aside $H=0$. Moreover, the constancy of the cost function in time- 
optimal problems makes that both primary constraint submanifolds are the same,

$$
N_{0}=N_{0}^{[0]}=N_{0}^{[-1]}=\left\{(\Lambda, u) \in T^{*}(T Q) \times U \mid \frac{\partial H}{\partial u^{k}}=H_{Y_{k}^{V}}=0, k=1, \ldots, m\right\},
$$

then $N_{1}=\left\{(\Lambda, u) \in T^{*}(T Q) \times U \mid H_{Y_{k}^{V}}=0, H_{\left[Z, Y_{k}^{V}\right]}=0, k=1, \ldots, m\right\}$, and so on until getting the final submanifold $N_{f}$, if it exists. Finally, the vanishing of the Hamiltonian must be considered to have the actual final constraint submanifolds

$$
\begin{gathered}
N_{f}^{[0]}=N_{f} \cap\left\{(\Lambda, u) \in T^{*}(T Q) \times U \mid H_{Z}+u^{k} H_{Y_{k}^{V}}=0\right\}, \\
N_{f}^{[-1]}=N_{f} \cap\left\{(\Lambda, u) \in T^{*}(T Q) \times U \mid H_{Z}+u^{k} H_{Y_{k}^{V}}=1\right\} .
\end{gathered}
$$

Due to condition (4.c) in Theorem 1, the zero fiber must be deleted from $N_{f}^{[0]}$.

Proposition 2. Let $\Sigma$ be an ACCS. Given a time-optimal control problem:

1. If $N_{f}^{[0]}$ has only zero covectors, there are no abnormal extremals.

2. If $N_{f}^{[0]}$ has nonzero covectors and $N_{f} \subset\left\{(\Lambda, u) \in T^{*}(T Q) \times U \mid\left(H_{Z}+u^{j} H_{Y_{j}^{V}}\right)=0\right\}$, then every abnormal extremal is strict and there are no normal extremals.

Remark 1. Indeed, Proposition 2 is also true for any control system. But in particular, for any control-affine system, fixed a point in $T Q$, the submanifold defined by the vanishing of the Hamiltonian is a subspace in the abnormality case.

\section{ACKNOWLEDGMENTS}

This work has been partially supported by the Spanish Ministerio de Educación y Ciencia, Project MTM2005-04947 and the Network Project MTM2006-27467-E/. MBL also acknowledges the financial support of the FPU grant AP20040096. We thank Professor Andrew D. Lewis and Professor David Martín de Diego for their useful ideas to develop this work. We also thank the anonymous referee for the useful comments.

\section{REFERENCES}

1. F. Bullo, A. D. Lewis, Geometric Control of Mechanical Systems. Modeling, analysis and design for simple mechanical control,Springer-Verlag, New York-Heidelberg-Berlin 2004.

2. J. F. CARiñenA, Theory of singular Lagrangians, Fortschr. Phys., 38(9)(1990), 641-679.

3. J. Cortés, S. Martínez, The consistency problem in optimal control, Reports on Math. Phys. 51 (2003), 171-186.

4. M. J. Gotay, J. M. Nester, Presymplectic Lagrangian systems I: The constraint algorithm and the equivalence theorem, Ann. Inst. H. Poincare Sect. A 30(2)(1979), 129-142.

5. W. LiU, H. J. Sussmann, Shortest paths for sub-Riemannian metrics on rank-two distributions, Mem. Amer. Math. Soc. 564, Jan. 1996.

6. C. López, E. MARTínez, Sub-Finslerian metric associated to an optimal control system, SIAM J. Control Optim 39(2000), 798-811.

7. R. Montgomery, Abnormal Minimizers, SIAM J. Control Optim., 32(6)(1994), 1605-1620.

8. L. S. Pontryagin, V. G. Boltyanski, R. V. Gamkrelidze, E. F. Mischenko, The Mathematical Theory of Optimal Processes, Interscience Publishers, Inc., New York 1962. 